\title{
Modal Analysis of Rotating Structures with Active Magnetic Bearing
}

\author{
Prince Owusu-Ansah ${ }^{1}$, Alex Frimpong Justice ${ }^{2}$, Philip Kwabena Agyemang ${ }^{3}$, \\ Saviour Kwame Woangbah ${ }^{4}$. \\ ${ }^{1}$ School of Mechanical and Electronic Engineering, Wuhan University of Technology \\ ${ }^{1}$ Mechanical Engineering Department, Kumasi Polytechnic P. O. Box 854, Kumasi Ghana \\ ${ }^{2}$ Mechanical Engineering Department, Kumasi Polytechnic P. O. Box 854, Kumasi Ghana \\ ${ }^{3}$ School of Mechanical and Electronic Engineering, Wuhan University of Technology,P.R China, 430070 \\ ${ }^{3}$ Mechanical Engineering Department, COE, KNUST, Kumasi Ghana \\ ${ }^{4}$ Mechanical Engineering Department, Kumasi Polytechnic P. O. Box 854, Kumasi Ghana.
}

Abstract: In this research paper a rotor which serves as a rotating structure is driven by a $4 K W$ alternating
current motor through couplings which is suspended byan active magnetic bearingwith its position Sensors and
Hall Effect Sensors connected in place which are finally connected to a main computer.
A digital link which interface between the DSP and the general mathematics program is made to run on
computer in which all the forces and the displacement signals are available in analog whichthen processed and
finally display the results. Natural frequencies and mode shapes were used for predicting dynamic behaviors of
rotor model, in determing this the free-free system were assumed without putting the bearings in operation and
then subsequently the rotational speed was set, the first three natural frequencies of the system without rigid
modes with its corresponding values was used as the process and stages observability and controllability of the
rotor system during the experiment and the analysis process.
Modal analysis was then performed for the free-free boundary conditions for the rotor system with bearing conditions when the rotor speed was equal to 20000 rpm.

The structure under investigation was established with a measured model input-output impulse response functions and the results studied and discussed.

Keywords: Active magnetic bearing, Modal analysis, Natural Frequency, Rotor, Rotor dynamic.

\section{Introduction}

Performing rotodynamic analysis in high speed rotating structures has gain more importance in recent times. The operation of high speed industrial rotating machines are very critical when taking into accounts its accompanying centrifugal effects such as high damping and its accompanied gyroscopic effect [1].

The interest in high speed rotors in various applications is increasing quickly, and, thanks to magnetic bearing technology and technical realization of such system has made it feasible for the use of components has resulted in the reduction of failures in this components [2]. Its important to note that the criterion for analyzing high rotor speed is very complex as used in mechanical design and the magnetic bearing control application which consists of variouscombination such as its geometry and also the number of its critical speeds that runs through the severity of external disturbances such as the unbalance forces are conditions that makes the design of an AMB use in high speed rotor system very demanding and a more complex task [3].

The use of rotor dynamic approach in analyzing these high speed rotating structure such as the rotor has the ability to identify parameters such as the damped and undamped values in addition to the various modes shape that are usual presented

Physical parameters are readily not available for theoretical derivations as stated in [4]. This is really in the case of high speed rotor designs which has complicated geometries such as shrink fits with additional masses and especially for many fluid structures which has interaction in squeeze film dampers, impellers, seals, fluid bearing etc. which are described by rotor dynamic coefficients in [5].

In such instance and situations, the appropriate required data will have to be taken into consideration from former experience and information which is available or will have to be determined by experimental process through identification procedures [6]. In identification techniques the main idea is mainly to excite the system which is normally under consideration with known input force and then to measure this input-output force relation to be able to identify the unknown system properties [7]. The main obstacle associated with when working with identification techniques in rotor dynamics is the excitation of the rotating system structure during its cycle of operation as captured in [8]. This is the case at one side it is very difficult to gain access to the rotating rotorand on the other side to the force measurement of the system is very difficult to measure 
Frequency response functions can be measured when vibrational properties are assessed, these can well be described in terms of modal parameters that is its natural frequencies, mode shapes and the damping coefficient, which together makes up a modal description of the rotating structure[9].

\section{Dynamic Characteristics Of Rotating Systems}

Generally, considering the equation of multiple-degrees-of-freedom systems.

$[\mathrm{M}]\{\ddot{D}\}+[\mathrm{C}]\{\dot{D}\}+[\mathrm{K}]\{\mathrm{D}\}=F$

Where $[D]=$ displacements vector for a problem of $n$ degree of freedom donated by $[\mathrm{Di}], \mathrm{i}=1,2 \ldots \mathrm{n}$.; $[\mathrm{F}]=$ external forces vector; $[\mathrm{M}]=$ mass matrix; $[\mathrm{C}]=$ damping matrix; and $[\mathrm{K}]$ is the stiffness matrix.

Equation (1) represents the governing equation of a transient structural simulation. The right hand side of the equation is the external force $[\mathrm{F}]$ and the first item of the left hand side of the equation, is inertia force, is damping force, and is the elastic force[10].

When analyzing the free vibration of a body there is no involvement of the external force [F]. So, equation (1), becomes

$[\mathrm{M}]\{\ddot{D}\}+[\mathrm{C}]\{\dot{D}\}+[\mathrm{K}]\{\mathrm{D}\}=0$

The equation (2) is used for a problem having $\mathrm{n}$ degrees of freedom. Our model has six degrees of freedom; it has at most six solutions of the fundamental natural frequency $(\omega)$. In a model analysis, we are interested in the natural frequencies and the relative shapes of the vibration modes[10]. However, the damping effect can be neglected in the equation (2) which results in equation (3).

$[\mathrm{M}]\{\ddot{\mathrm{D}}\}+[\mathrm{K}]\{\dot{\mathrm{D}}\}=0$

Equation (3) is used in during modal analysis system to solve for the natural frequency for rotor model used in rotating structures.

When using a stationery reference frame, the reference analysis system is attributed to the global coordinate system, which is a fixed. In such analysis the system, gyroscopic moments due to nodal rotations are included in the damping matrix.

Determining the physical M, D, K canalways be done by calculation or by measurement. This procedure is refer as identification, precisely when the structure of the rotor dynamic model is known as stated in equation (1)From the measured input and output signal, the dynamic characteristic is calculated by means of known and output relation using time and or frequency domain

\section{Frequency Response Functions}

This process of measurement is to excite the rotor dynamic system by artificially or kinematic excitation, this ensures that the input and output signals are measured, and the process function are then used later during the parameters estimation[11].

Equation (4) and (5) shows out the main relationship that exists between the measuring compliance function that is the (force excitation) and the stiffness function also known as the kinematic excitation. The compliance $\bar{H}_{k l}(\omega)$ is described as the out $\hat{\bar{Z}}_{k}(\omega)$ which is divided by the force of excitation $\hat{\bar{f}}_{l}(\omega)$, in which all the other forces are considered as zero. Considering the other hand of the relation $\bar{K}_{k l}(\omega)$ is found from the force $\hat{\bar{f}}_{k}$ that will have to be applied to the sytem in which only the displacement $\hat{\bar{Z}}_{l}(\omega)$ is deem to be present. This shows why it is easier to measure the compliance rather than the stiffness, so it is easier to apply only a single force and practically impossible to constrain all the system with one degree of freedom $\bar{H}(\omega)$ and $\bar{K}(\omega)$ which are usually nonsymmetrical matrices, due to the possible nonsymmetry in $K$ and $D$.

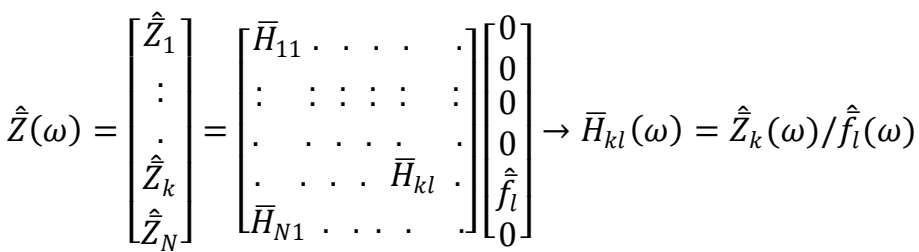

To calculate the amplitude of the compliance function $\bar{H}_{k l}(\omega)$, the system is excited by force amplitude $\hat{\hat{f}_{l}}$ only and the response $\hat{\bar{Z}}_{k}$ is measured. $\bar{H}_{k l}$ can then be determined from the ratio $\hat{\bar{Z}}_{k} / \hat{\bar{f}}_{l}$.

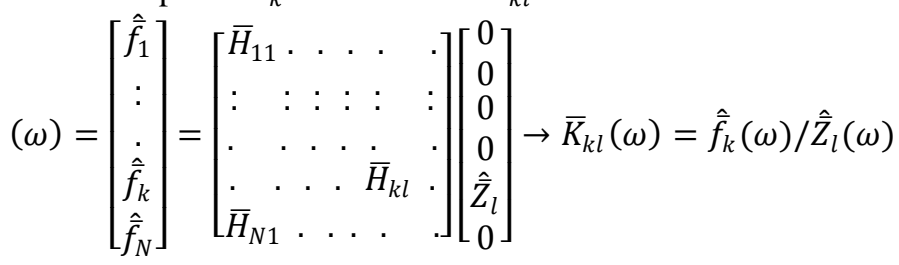


In a similar situation, $\bar{K}_{k l}(\omega)$ is found by the ratio of $\hat{\bar{f}}_{k} / \hat{\bar{Z}}_{l}$ when the system is deem to be excited only by $\hat{\bar{Z}}_{l}$, and the force $\hat{\bar{f}}_{k}$ is then measured.

Different kinds of devices developed for excitation have been carried out for the purpose of identification in recent times, either for the purpose of real machinery or for small test rigs. These include preloading of a shaft with a snap back, hammer impact method as well as unbalance excitation through a second shaft with different running speeds, shaking of the shaft through a rider and the use of active magnetic bearing.

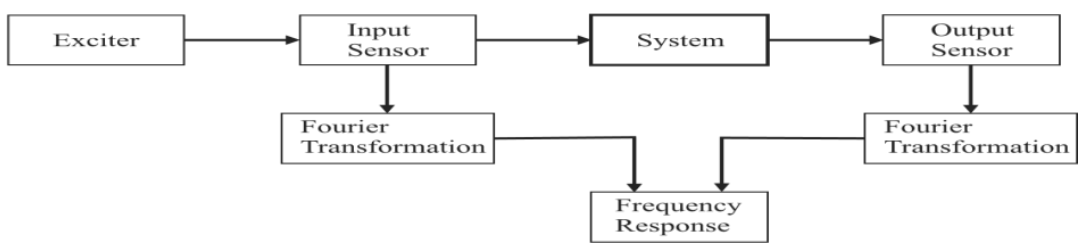

Fig. 1 Configuration to measure frequency response functions

\section{Analysis of Rotating Structure}

A rotor which is driven by a $4 \mathrm{KW}$ alternating current motor (AC) through couplings in fig 2 shows the various AMBs (AMB1, AMB2) with their Position Sensors (PS) and Hall Effect Sensors connected in place, all this component eventually are linked to the main PC and is controlled by mathematic program MATLAB which allows detection of the mode shapes of the structure to be done.

The main membrane coupling which works like a cardiac joint allows the shaft ends to move in a radial and tilting displacement directions with a defined low stiffness.

Fig. 2 shows a photograph of the test rig configuration with a rigid disk mounted on a heavy concrete platform which has been decoupled from the environment with viscous dampers.The foundation of the rigid body modes of the test rig are all below $14 \mathrm{~Hz}$ and this is far away from the first bending eigenfrequency of the rotor.

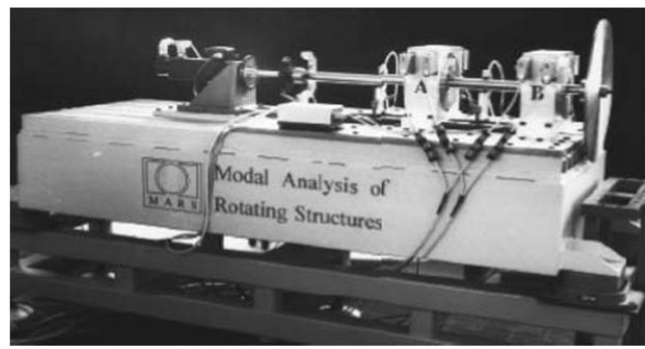

Fig. 2 Photograph of the test rig configuration.

\section{Natural Frequencies And Mode Shapes Analysis}

Natural frequencies and mode shapes are very important for predicting dynamic behaviors of rotor model.In determing this we start with the free-free system in Fig. 3 assuming the system are without bearings and the rotational are set to zero. The first three natural frequencies of the system without rigid modes with its corresponding values are indicated in Fig. 3 as $50 \mathrm{~Hz}, 130 \mathrm{~Hz}$, and $355 \mathrm{~Hz}$ which also indicates the positions of the actuators and sensors. This informationwas used for the evaluation of the observability and the controllability of the rotor system.

The calculated mode shapes and natural frequencies were used as reference points for the measurement for the rotor system during the experiment.

The experimental modal analysis was then performed for the free- free boundary conditions for the rotor system when the rotor speed was equal to zero. The calculated and measured natural frequencies were in good correlation as could be seen in fig. 3 of the first three natural frequencies of the free-free rotor system which confirms that the model was in good condition and can be used for further purpose. In reality, it is considered that the boundary conditions are not free as stated by [13,14]. Electromagnet forces are used to support the active magnetic bearing which is dependent on the control current in the windings and on the air gap which is has been discussed in [15]. The second case of the rotor system with bearing stiffness of kwas considered during the analysis which depended on the control parameters, for the operational point of the system. The stiffness kwas calculated and then used for the calculation of the natural frequencies of the elastically supported shaft. 


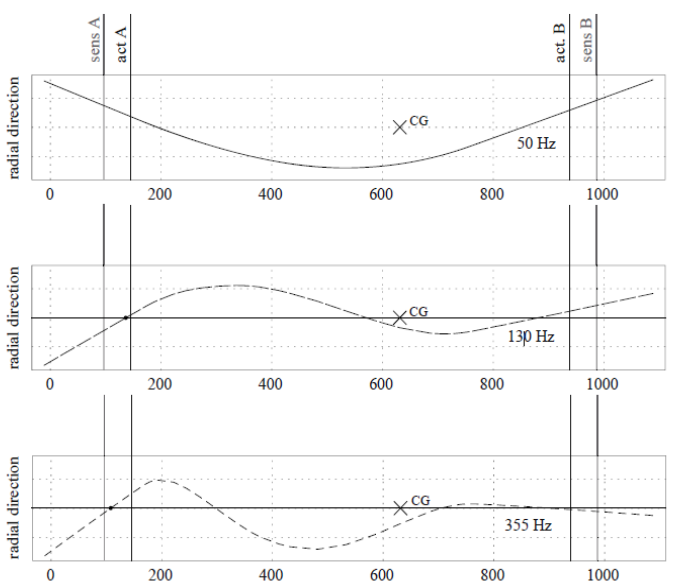

Axial position along the shaft/mm

Fig. 3.The three first natural frequencies of the free-free rotor system

The bearing stiffness calculated for the active control model was $\mathrm{k}=1000 \mathrm{~N} / \mathrm{mm}$ for the controller with low implication band. The rotational speed was still assumed to be zero. The natural frequencies for this system which includes new modes were $33 \mathrm{~Hz}, 57 \mathrm{~Hz}, 66 \mathrm{~Hz}$, and $131 \mathrm{~Hz}$, which is indicated in Fig. 4, the resultant is the five natural frequencies compared to three in the previous case, and this is obviously due to the increase in the number for the model modes. Changes in bearing stiffness which occurs due to the control current stiffness parameter $\mathrm{k}$ was varied in a wide range during the analysis and it was obvious that by increasing bearing stiffness $\mathrm{k}$, the natural frequencies to $50 \mathrm{~Hz}, 130 \mathrm{~Hz}$ and $355 \mathrm{~Hz}$ as shown in Fig. 3 compared to the original 0 frequency of the rigid mode shape which became $33 \mathrm{~Hz}$ and $57 \mathrm{~Hz}$, with its mode shapes shown in Fig. 4.
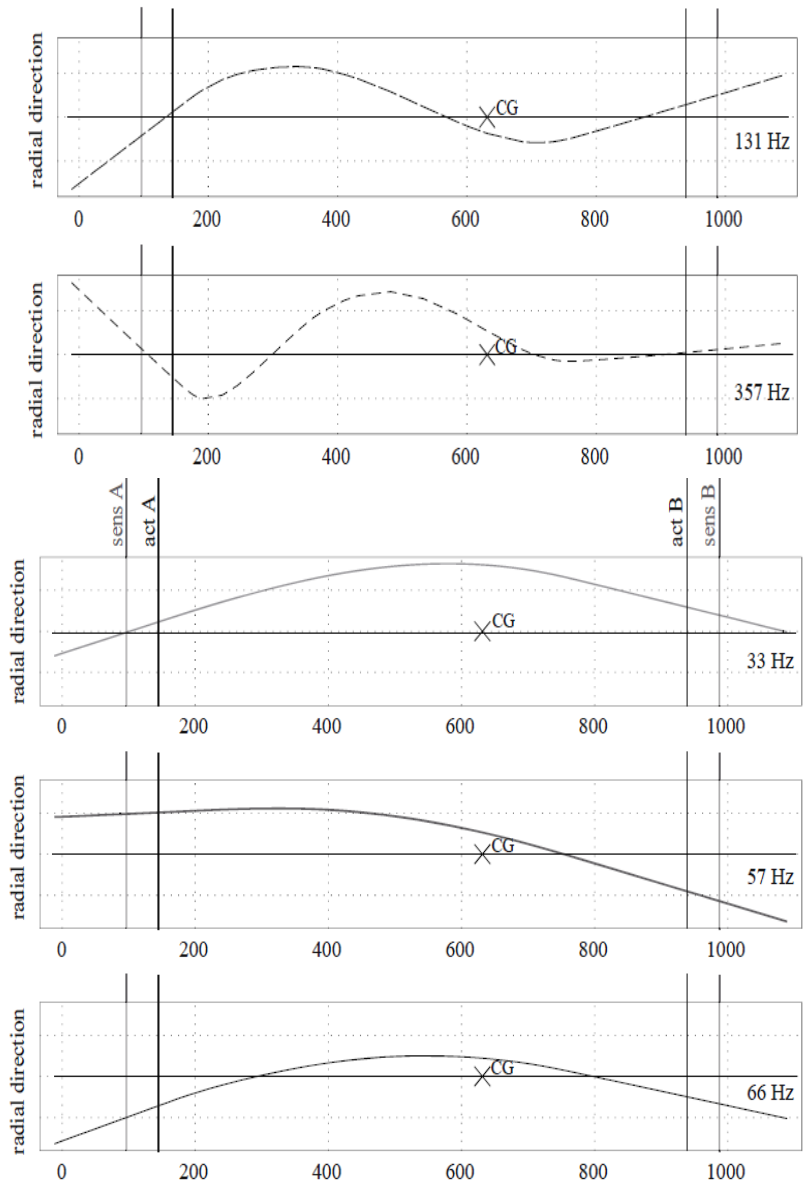

Axial position along the shaft $/ \mathrm{mm}$

Fig 4. The five natural frequencies of the rotor system with bearing stiffness 
The new mode shape are now characterized by rigid motion which are superimposed by additional bending. The natural frequencies were $66 \mathrm{~Hz}(50 \mathrm{~Hz}), 131 \mathrm{~Hz}(130 \mathrm{~Hz})$ and $357(355 \mathrm{~Hz})$.

It could be observed that higher modes actually do not changes much with increasing bearing stiffness.

Increasing the bearing stiffness will further results in the natural frequencies also becoming higher with its changing modes, finally when the bearing stiffness are very high, the mode shapes are also been characterized by zero displacement at the bending locations which corresponds to the clamp boundary conditions.

\section{Rotor Excitation With Active Magnetic Bearing}

Fig. 5 describes a schematic diagram of an AMB exciter system. In this system, a position control is needed to levitate the rotor along with the magnetic bearings a digital process (DSP) is made to run the control program with a sampling time of $440 \mu$ s. It was also used to compute the force from the measured flux and its position signals.

A force and a position signal are acquired on the DSP. A digital link which interface between the DSP and the general mathematics program MATLAB is made to run on a personal computer. In this process the force and the displacement signals are made available in the analog which is then processed together with the signal from the additional sensors.

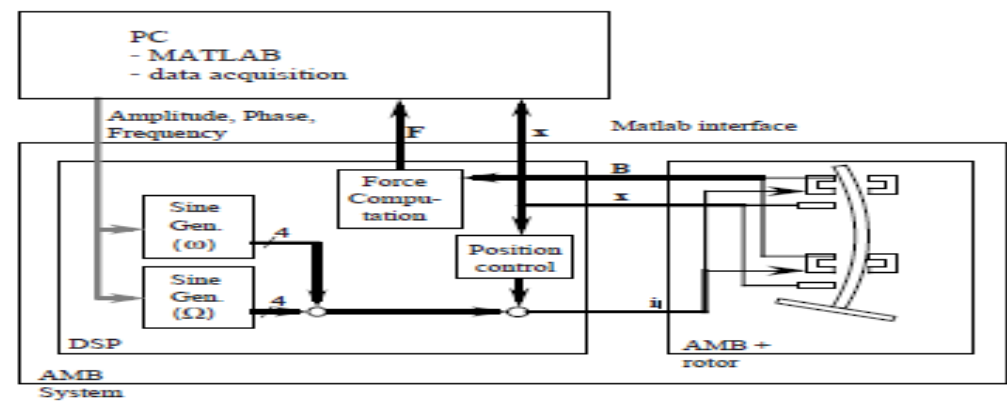

Fig. 5Schematic of AMB Exciter System.

During the excitation process two sinewave generator are implemented on the DSP one of them is synchronized to the rotors revolution speed, whiles the other is used as a user identifier. Each of the sinewaves generator consists of a four output signals which are then connected to the four control currents of the $\mathrm{x}$ and $\mathrm{y}$ directions of the two bearings.

The amplitude and the relative phases of each output are then defined through MATLAB, exciting the rotating system structure artificially during its period of operation and then measuring the system excitation and then this measured input and output signal are then calculated by means of existing known input and output relationship in the frequency and time domain.

Which is based on this model of input-output functions as well as impulse response functions (time domain) or frequency response function, frequency domain can now be a calculated, as the system parameters are now assumed, these functions are also determined from the measured input and output signal using the signal processors.

\section{Conclusion}

Modal analysis of the rotating structure has been performed for the free- free boundary conditions for the rotor system. The calculated and measured natural frequencies were in good correlation as could be seen in Fig. 3 of the first three natural frequencies of the free-free rotor system.

The second case of the rotor system with bearing stiffness of kwas considered during the analysis which depended on the control parameters, for the operational point of the system. The stiffness kwas calculated and then used for the calculation of the natural frequencies of the elastically supported shaft.

The bearing stiffness calculated for the active control model was $\mathrm{k}=1000 \mathrm{~N} / \mathrm{mm}$ for the controller with low implication band as the rotational speed was still assumed to be zero. The natural frequencies for the new system which included new modes were $33 \mathrm{~Hz}, 57 \mathrm{~Hz}, 66 \mathrm{~Hz}$, and $131 \mathrm{~Hz}$ are indicated in fig. 4 , the resultant is the five natural frequencies modes shapes indicated in Fsig.4), as compared to the three modes shapes as shown previously in (fig.3), the effect in changes of rapid modes is as a result in the increase in the number for the frequency modes.

Changes in bearing stiffness which occurs due to the control current stiffness parameter $\mathrm{k}$ was varied in a wide range during the analysis and it was obvious that by increasing bearing stiffness kresulted in the natural 
frequencies to increases to $50 \mathrm{~Hz}, 130 \mathrm{~Hz}$ and $355 \mathrm{~Hz}$ as shown in (fig.3), compared to the original 0 frequency of the rigid mode shape which became $33 \mathrm{~Hz}$ and $57 \mathrm{~Hz}$, with its mode shapes shown in (fig.4).

The new mode shape which were characterized by rigid motion are was superimposed by additional bending of natural frequencies of $66 \mathrm{~Hz}(50 \mathrm{~Hz}), 131 \mathrm{~Hz}(130 \mathrm{~Hz})$ and $357(355 \mathrm{~Hz})$.

It could be observed that higher modesshape actually do not changes much with increasing bearing stiffness.

Increasing the bearing stiffness will further results in the natural frequencies also becoming higher with its changing modes as the bearing stiffness becomes are very high resulting in the mode shapes are also been characterized by zero displacement at the bending locations which corresponds to the clamp boundary conditions.

\section{Acknowledgements}

This work was supported by The Natural Science Foundation of China (NO.51275372) and Wuhan High-Tech Development Project Foundation (NO.201110921299) and The Fundamental Research Funds for the Central Universities (Wuhan University of Technology No.2012-IV-036).

\section{References}

[1]. E. Knopf, and R. Nordmann, Identification of the Dynamic Characteristics of Turbulent, Journal Bearings using Active Magnetics Bearings. In 7th International Conference on Vibration in Rotating Machinery, 56(8), 2000, 143-148.

[2]. M. Aenis, and R. Nordmann, Active magnetic Bearings for Fault Detection in a Centrifugal Pump. In 7th International Symposium on Magnetic Bearings, 33(15), 2000, 276-280.

[3]. E.Knopf, and R. Nordmann, Active Magnetic Bearings for the Identification of Dynamic Characteristics of Fluid Bearings, In 6th International Symposium on Magnetic Bearings, 12(5), 1998, 102-109.

[4]. R. A.Larsonneur,High Speed Rotor in Active Magnetic Bearings. In the Proceedings of the 3rd International Conference on Rotordynamics, 5(8), 1990, 201-209.

[5]. M.Hirschmanner, and H. Springer, Adaptive Vibration and Unbalance Control of a Rotor Supported by Active Magnetic Bearing. In Proceedings of the 8th International Symposium on Magnetic Bearings, 10(9), 2002, 321-326.

[6]. R. Larsonneur, R.Stewart, and A. Traxler, A. Active Magnetic Bearing Control of Magnetic Bearing Systems with Imbalance. In the proceedings of the 5th International Symposium on Magnetic Bearings, 8(9), 2008, 344-354

[7]. Kirk, Rotor Drop Test Stand for AMB Rotating Machinery Part II: Steady State Analysis and Comparison to Experimental Results, Proceedings of Fourth Int. Sym. on Magnetic Bearings, 12 (10), 1994, 276-282.

[8]. K. Adler, C.H. Schalk, and R. Nordamann, Active Balancing of a Super Critical Rotor onActive Magnetic Bearing,Tenth International Symp. On Magnetic Bearings, 25(23), 2006, 49-54.

[9]. F.W Fomi, (2008). Active Balancing of a Flexible Rotor in Active Magnetic Bearings, Fifth International Symposium. On Magnetic Bearing, 5(12), 2008, 65-78.

[10]. Shiyu, Z. (2001). Active Balancing and Vibration Control of Rotating Machinery, Fifth International Conference of Survey, Shock and Vibrational Digest, 33(5), 361-371.

[11]. L.Guoxin, M. Eric, and A. Paul, A note on ISO AMB- Rotor System Stability Margin, In proceedings of the Tenth International Symposium on Magnetic Bearings, 14(16),2006,156-167.

[12]. E.H.Maslen, and J.R. Bielk, A Stability model for Flexible Rotors with Magnetic Bearings. ASME Journal of Dynamic System Measurement, and Control, 114(1),2008, 172-175.

[13]. D.Meekers, and E. Maslen, Analysis and Control of a Three Radial Magnetic Bearings. Proceedings of the Tenth International Symposium on Magnetic Bearing, 25(30), 2010, 231-138.

[14]. L. Hawkins, P. McMullen, and R. Larsonneur, Development of an AMB Energy Storage Flywheel for Commercial Application. In International Symposium on Magnetic Suspension Technology, 8(12), 2005, 212-221.

[15]. G. Schweitzer, (2002) Active Magnetic Bearings-Chances and Limitation.6th International Conference on Rotor Dynamics Analysis, 15(17), 2002, 34-39. 